\title{
AROUND A CENTRAL ELEMENT OF A NEARLATTICE
}

\author{
Jahanara Begum \\ Department of Mathematics, Dhaka College, Dhaka \\ Email :drk_azam@yahoo.com \\ and \\ A.S.A. Noor \\ Department of ECE, East West University, Dhaka. \\ E mail: noor@ewubd.edu
}

Received 30.12.2010

Accepted 25.02.2012

\begin{abstract}
A nearlattice $S$ is a meet semilattice together with the property that any two elements possessing a common upper bound have a supremum. It is well known that if $n \in S$ is a neutral and upper element then its isotope $S_{n}=(S ; \cap)$ is again a nearlattice, where $x \cap y=(x \wedge y) \vee(x$ $\wedge n) \vee(y \wedge n)$ for all $x, y \in S$. In this paper we have discussed the central elements in a nearlattice and also in a lattice. We included several characterizations of these elements. We showed that for a central element $n \in S, P_{n}(S) \cong(n]^{d} \times[n)$, where $P_{n}(S)$ is the set of principal n-ideals of $S$. Then we proved that for a central element $n \in S$, an element $t \in S$ is central if and only if it is central in $S_{n}$. We also proved that for a lattice $L, L_{n}$ is again a lattice if and only if $n$ is central. Finally we showed that $B$ is a Boolean algebra if and only if $B_{n}$ is a Boolean algebra with same complement when $n$ is central. Moreover, $B \cong B_{n}$.
\end{abstract}

Keywords: Central element, Nearlattice, Isotope, Boolean algebra.

\section{Introduction}

By a nearlattice $S$, we will always mean a (lower) semilattice which has the property that any two elements possessing a common upper bound, have a supremum. Nearlattice will form a lattice if it has a largest element. A nearlattice $S$ is distributive if and only if for all $x, y, z \in S, t \wedge((x \wedge y) \vee(x \wedge z)=(t \wedge x \wedge y) \vee(t \wedge x \wedge z)$. Let $S$ be a nearlattice and $S \in S$. Then $S$ is called a standard element if for all $x, y, t \in S, t \wedge[(x \wedge y) \vee(x \wedge s)]=(t \wedge x \wedge y)$ $\vee(t \wedge x \wedge s)$. In a nearlattice, an element $s$ is neutral if for any $t, x, y \in S, s$ is standard and $s \wedge[(t \wedge x) \vee(t \wedge y)]=(s \wedge t \wedge x) \vee(s \wedge t \wedge y)$. An element $s$ of a nearlattice $S$ is called a medial element if $\mathrm{m}(x, s, y)=(x \wedge y) \vee(x \wedge s) \vee(y \wedge s)$ exists for all $x, y \in S$. An element $s$ of a nearlattice $S$ is called sesquimedial if for all $x, y, z \in S, J_{s}(x, y, z)$ exists in $S$ where $J_{s}(x, y, z)=[(x \wedge s) \vee(y \wedge s)] \wedge[(y \wedge s) \vee(z \wedge s)] \vee(x \wedge y) \vee(y \wedge z)$. Every sesquimedial element is medial. An element $n$ of a nearlattice $S$ is called an upper element if $x \vee n$ exists for all $x \in S$. Every upper element is of course sesquimedial. An element is called a central element of $S$ if it is neutral, upper and complemented in each interval containing it. We know by [2] that for a neutral element $n \in S$ if $n$ is sesquimedial then $S_{n}=(S ; \cap)$ is again a nearlattice where $x \cap y-(x \wedge y) \vee(x \wedge n) \vee(y \wedge n)$. For a fixed element $n$ of a nearlattice 
$S$, a convex subnearlattice containing $n$ is called an $n$-ideal. An $n$-ideal generated by a finite number of elements $a_{1}, \ldots, a_{m}$ is called a finitely generated n-ideal denoted by $\left(a_{1}, \ldots\right.$, $\left.a_{m}\right)_{n}$. Set of all finitely generated $n$-ideals of $S$ is denoted by $F_{n}(S)$. An $n$-ideal generated by a single element is called a principal $n$-ideal. The set of all principal $n$-ideals of $S$ is denoted by $P_{n}(S)$. If $S$ is a lattice then $\left(a_{1}, \ldots, a_{m}\right) n=\left[a_{1} \wedge \ldots \wedge a_{m} \wedge n, a 1 \vee \ldots \vee a_{m} \vee n\right]$. Thus $(a)_{n}=[a \wedge n, a \vee n]$. For detailed literature on $n$-ideal of lattices and nearlatices we refer the reader to consult [3], [5], [7], [8]. In this paper we have given several characterizations of central elements of a nearlattice. We proved that for a central element $n \in S, P_{n}(S) \cong(n]^{d} \times[n)$. Then we proved that for a central element $n \in S$, an element $t \in S$ is central if and only if it is central in $S_{n}$. We also showed that for a lattice $L, L_{n}$ is again a lattice if and only if $n$ is central. Finally we extended a result of Goetz's result on isotopes of Boolean algebras.

\section{Isotopes $L_{n}$ when $n$ is a Central Element}

We start this paper with the following characterization of a central element of a nearlattice.

Theorem 1.1. Let $S$ be a nearlattice and $n \in S$. Then the following conditions are equivalent :

(i) $n$ is central;

(ii) $n$ is standard, upper and complemented in each interval containing it.

Proof. (i) $\Rightarrow$ (ii) is trivial from the definition .

(ii) $\Rightarrow$ (i). Suppose $n$ is standard and complemented in each interval containing it. It is enough to prove that $n \wedge((t \wedge x) \vee(t \wedge y)=(n \wedge t \wedge x) \vee(n \wedge t \wedge y)$

Since $\quad(n \wedge t \wedge x) \vee(n \wedge t \wedge y) \leq n \leq(t \wedge x) \vee(t \wedge y) \vee n$, there exists $r \in S$ such that $n \wedge r=(n \wedge t \wedge x) \vee(n \wedge t \wedge y)$ and $n \vee r=(t \wedge x) \vee(t \wedge y) \vee n$

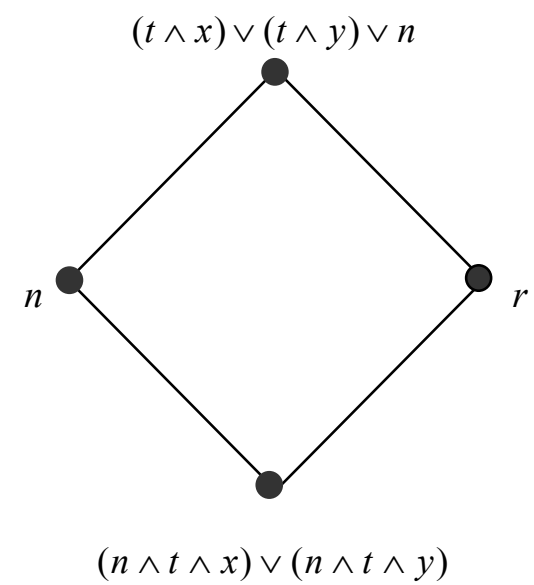

Figure 1 
Now $t \wedge x=(t \wedge x) \wedge[(t \wedge x) \vee(t \wedge y) \vee n]$

$$
\begin{aligned}
& =(t \wedge x) \wedge(n \vee r) \\
& =(t \wedge x \wedge n) \vee(t \wedge x \wedge r)(\text { as } n \text { is standard })
\end{aligned}
$$

Similarly $t \wedge y=(t \wedge y \wedge n) \vee(t \wedge y \wedge r)$

So $(t \wedge x) \vee(t \wedge y)=(t \wedge x \wedge n) \vee(t \wedge x \wedge r) \vee(t \wedge y \wedge n) \vee(t \wedge y \wedge r)$

$$
=(t \wedge x \wedge r) \vee(t \wedge y \wedge r) \vee(n \wedge r) \leq r
$$

Therefore $n \wedge((t \wedge x) \vee(t \wedge y)) \leq n \wedge r$

$$
=(n \wedge t \wedge x) \vee(n \wedge t \wedge y)
$$

But the reverse inequality is trivial.

Hence $n \wedge((t \wedge x) \vee(t \wedge y))=(n \wedge t \wedge x) \vee(n \wedge t \wedge y)$

Therefore $n$ is neutral, and so $n$ is central $\square$

We know from [1] that for a neutral element $n$ of a lattice $L, L_{n}$ is a medial nearlattice. Now we have the following result.

Theorem 1.2. Suppose $L$ is a lattice and $n \in L$ is standard. Then the isotope $L_{n}$ is a lattice if and only if $n$ is central in $L$.

Proof. Since $n$ is standard, so by [2, Theorem 2.1]

$$
(L ; \subseteq) \cong\left(P_{n}(L) ; \subseteq\right)
$$

Thus $L_{n}$ is a lattice if and only if $\left(P_{n}(L) ; \subseteq\right)$ is a lattice, But by [7],

$P_{n}(L)$ is a lattice if and only if $n$ is complemented in each interval containing it. Therefore $L_{n}$ is a lattice if and only if $n$ is central in $L \square$

Corollary 1.3. For a central element $n \in L$ of a bounded lattice, $L_{n}$ is also a bounded lattice with $n$ as the smallest and $n^{\prime}$ as the largest element.

Moreover, $x \cap y=m(x, n, y)$ and $x \cup y=m\left(x, n^{\prime}, y\right)$

Theorem 1.4. Let $L$ be a bounded lattice and $n \in L$ be central. If $n^{\prime}$ is the complement of $n$ then $n^{\prime}$ is also central.

Proof. Let $a \leq n^{\prime} \leq b$

Consider $(a \vee n) \wedge b$ 
Now $n^{\prime} \wedge[(a \vee n) \wedge b]=\left[n^{\prime} \wedge(a \vee n)\right] \wedge b$

$$
\begin{aligned}
& \left.=\left[\left(a \wedge n^{\prime}\right) \vee\left(n \wedge n^{\prime}\right)\right] \wedge b \text { (as } n \text { is standard }\right) \\
& =\left[\left(a \wedge n^{\prime}\right) \vee 0\right] \wedge b \\
& =a \wedge n^{\prime} \wedge b=a \\
n^{\prime} \vee[(a \vee n) \wedge b]= & n^{\prime} \vee(a \wedge b) \vee(b \wedge n) \quad(\text { as } n \text { is standard }) \\
& =n^{\prime} \vee a \vee(b \wedge n) \\
& =n^{\prime} \vee(b \wedge n) \\
& =\left(b \wedge n^{\prime}\right) \vee(b \wedge n) \\
& =b \wedge\left(n \vee n^{\prime}\right) \quad(\text { as } n \text { is standard }) \\
& =b \wedge 1=b .
\end{aligned}
$$

Therefore $(a \vee n) \wedge b$ is the complement of $n^{\prime}$ in $[a, b]$.

Now we shall show that for all $x, y \in L$,

$x \wedge\left(y \vee n^{\prime}\right)=(x \wedge y) \vee\left(x \wedge n^{\prime}\right)$

Now $n \wedge\left[x \wedge\left(y \vee n^{\prime}\right)\right]=x \wedge n \wedge\left(y \vee n^{\prime}\right)$

$$
\begin{aligned}
& =x \wedge\left[(y \wedge n) \vee\left(n \wedge n^{\prime}\right)\right](\text { as } n \text { is neutral }) \\
& =x \wedge y \wedge n
\end{aligned}
$$

Also $n \wedge\left[(x \wedge y) \vee\left(x \wedge n^{\prime}\right)\right]=(x \wedge y \wedge n) \vee\left(x \wedge n \wedge n^{\prime}\right)$

$$
=x \wedge y \wedge n \quad \text { (as } n \text { is neutral) }
$$

Again $n \vee\left[x \wedge\left(y \vee n^{\prime}\right)\right]=(n \vee x) \wedge\left(y \vee n \vee n^{\prime}\right)=n \vee x$,

$$
\text { and } \begin{aligned}
n \vee\left[(x \wedge y) \vee\left(x \wedge n^{\prime}\right)\right] & =(x \wedge y) \vee n \vee\left(x \wedge n^{\prime}\right) \\
& =(x \wedge y) \vee\left[(n \vee x) \wedge\left(n \vee n^{\prime}\right)\right],(\text { as } n \text { is distributive }) \\
& =(x \wedge y) \vee[(n \vee x) \wedge 1] \\
& =(x \wedge y) \vee(n \vee x) \\
& =n \vee x
\end{aligned}
$$

Therefore $x \wedge\left(y \vee n^{\prime}\right)=(x \wedge y) \vee\left(x \wedge n^{\prime}\right)$, (as $n$ is standard $)$.

So $n^{\prime}$ is standard. 
Therefore $n^{\prime}$ is central by Theorem 1.1.

The following result is due to Kolibiar [6]

Lemma 1.5. If an element is central in a lattice then it is also central in the dual lattice.

Proof. Let $n$ be central in $L$. Suppose $a \leq_{d} n \leq_{d} b$ in $L^{d}$.

Then $b \leq n \leq a$ in $L$.

So there exists $t \in[b, a]$ such that $n \wedge t=b$ and $n \vee t=a$ in $L$.

Then $n \vee_{d} t=b$ and $n \wedge_{d} t=a$ in $L^{d}$.

Thus $n$ is complemented in $[a, b]$ in $L^{d}$.

Moreover for all $x, y \in L^{d}, x \wedge_{d}\left(y \vee_{d} n\right)=x \vee(y \wedge n)=x \vee(x \wedge n) \vee(y \wedge n)$

$=x \vee[n \wedge(x \vee y)]($ as $n$ is neutral $)$,

$=[(x \vee y) \wedge x] \vee[(x \vee y) \wedge n]$

$=(x \vee y) \wedge(x \vee n)($ as $\mathrm{n}$ is standard $)$,

$=\left(x \wedge_{d} y\right) \vee_{d}\left(x \wedge_{d} n\right)$.

This implies $n$ is standard in $L^{d}$.

Therefore by Theorem 1.1, $n$ is neutral and hence central in $L^{d} \square$

Now we give a characterization of central element in a nearlattice.

Lemma 1.6. Suppose $S=A \times B$ where $A$ is a lattice and $B$ is a nearlattice. Then any element $t=\left(t_{1}, t_{2}\right)$ of $S$ is central if and only if $t_{1}, t_{2}$ are central in $A$ and $B$ respectively.

Proof. Suppose $t=\left(t_{1}, t_{2}\right)$ is central in $S$.

Let $p_{1} \leq t_{1} \leq q_{1}$ in $A$. Then $\left(p_{1}, t_{2}\right) \leq\left(t_{1}, t_{2}\right) \leq\left(q_{1}, t_{2}\right)$

Then there exists $\left(r_{1}, r_{2}\right) \in S$ such that $\left(t_{1}, t_{2}\right) \wedge\left(r_{1}, r_{2}\right)=\left(p_{1}, p_{2}\right)$ and $\left(t_{1}, t_{2}\right) \vee\left(r_{1}, r_{2}\right)=\left(q_{1}, t_{2}\right)$.

This implies $r_{1} \wedge t_{1}=p_{1}$ and $r_{1} \vee t_{1}=q_{1}$.

So $t_{1}$ is complemented in each interval containing it in $A$.

For $x, y \in A,\left(x, t_{2}\right) \wedge\left[\left(y, t_{2}\right) \vee\left(t_{1}, t_{2}\right)\right]$

$=\left(\left(x, t_{2}\right) \wedge\left(y, t_{2}\right)\right) \vee\left(x, t_{2}\right) \wedge\left(t_{1}, t_{2}\right),\left(\right.$ as $\left(t_{1}, t_{2}\right)$ is standard in $\left.S\right)$.

This implies $\left(x \wedge\left(y \vee t_{1}\right), t_{2}\right)=\left((x \wedge y) \vee\left(x \wedge t_{1}\right), t_{2}\right)$, 
Then $x \wedge\left(y \vee t_{1}\right)=(x \wedge y) \vee\left(x \wedge t_{1}\right)$ and so $t_{1}$ is standard in $A$.

Thus $t_{1}$ is central in $A$.

Similarly $t_{2}$ is central in $B$.

Conversely, Let $t_{1}, t_{2}$ be central in $A$ and $B$.

Let $\left(p_{1}, p_{2}\right) \leq\left(t_{1}, t_{2}\right) \leq\left(q_{1}, q_{2}\right)$.

This implies $p_{1} \leq t_{1} \leq q_{1}$ and $p_{2} \leq t_{2} \leq q_{2}$.

So there exists $r_{1} \in A, r_{2} \in B$, such that,

$p_{1}=r_{1} \wedge t_{1}, p_{2}=r_{2} \wedge t_{2}, q_{1}=r_{1} \vee t_{1}$ and $q_{2}=r_{2} \vee t_{2}$

Hence $\left(t_{1}, t_{2}\right) \wedge\left(r_{1}, r_{2}\right)=\left(p_{1}, p_{2}\right)$ and $\left(t_{1}, t_{2}\right) \vee\left(r_{1}, r_{2}\right)=\left(q_{1}, q_{2}\right)$

Therefore $\left(r_{1}, r_{2}\right)$ is the relative complement of $\left(t_{1}, t_{2}\right)$ in

$\left[\left(p_{1}, p_{2}\right),\left(q_{1}, q_{2}\right)\right]$.

Again for $(x, y),(p, q) \in S$,

$$
\begin{aligned}
(x, y) \wedge[ & \left.(p, q) \vee\left(t_{1}, t_{2}\right)\right]=\left(x \wedge\left(p \vee t_{1}\right), y \wedge\left(q \vee t_{2}\right)\right) \\
& \left.=\left((x \wedge p) \vee\left(x \wedge t_{1}\right),(y \wedge q) \vee\left(y \wedge t_{2}\right)\right) \quad \text { as } t_{1}, t_{2} \text { are standard }\right), \\
& =(x \wedge p, y \wedge q) \vee\left(x \wedge t_{1}, y \wedge t_{2}\right) \\
& =((x, y) \wedge(p, q)) \vee\left((x, y) \wedge\left(t_{1}, t_{2}\right)\right)
\end{aligned}
$$

This implies $\left(t_{1}, t_{2}\right)$ is standard in $S$ and hence it is central $\square$

Thus we have the following result .

Corollary 1.7. For lattices $A$ and $B$ in $L=A \times B$ an element $t=\left(t_{1}, t_{2}\right) \in A \times B$ is central if and only if $t_{1}$ and $t_{2}$ are central in $A$ and $B$ respectively $\square$

Lemma 1.8. For a central element $n$ of a nearlattice $S, S_{n} \cong(n]^{d} \times[n)$.

Proof. Consider the map $\varphi: S_{n} \rightarrow(n]^{d} \times[n)$, defined by $\varphi(a)=(a \wedge n, a \vee n)$

Suppose $a \leq b$ in $S_{n}$. Then $a=(a \wedge b) \vee(a \wedge n) \vee(b \wedge n)$

and so $b \wedge n \leq a \wedge n \leq a \vee n \leq b \vee n$.

Thus $a \wedge n \leq_{d} b \wedge n$ in $(n]^{d}$ and $a \vee n \leq b \vee n$ in $[n)$.

Hence $(a \wedge n, a \vee n) \leq(b \wedge n, b \vee n)$ in $(n]^{d} \times[n)$

Therefore $\varphi$ is isotone (order preserving). 
Now let $a, b \in S_{n}$ are such that $\varphi(a) \leq \varphi(b)$

That is $(a \wedge n, a \vee n) \leq(b \wedge n, b \vee n)$ in $(n]^{d} \times[n)$.

Then $a \wedge n \leq_{d} b \wedge n$ in $(n]^{d}$ and $a \vee n \leq b \vee n$ in $[n)$.

This implies $b \wedge n \leq a \wedge n \leq a \vee n \leq b \vee n$ in $S$.

So $\langle a\rangle_{n} \subseteq\langle b\rangle_{n}$.

Thus by [2, Theorem 2.1], $a \subseteq b$ in $S_{n}$

and this says that $\varphi$ is order isomorphism.

Finally, Let $\left(t_{1}, t_{2}\right) \in(n]^{d} \times[n)$.

Then $t_{1} \leq n \leq t_{2}$ since $n$ is central,

so there exists $c \in S$, such that $t_{1}=c \wedge n, t_{2}=c \vee n$ implies

$\left(t_{1}, t_{2}\right)=(c \wedge n, c \vee n)=\varphi(c)$, implies $\varphi$ is onto.

Therefore $\varphi$ is isomorphism

Now we include another characterization of a central element in a nearlattice.

Lemma 1.9. Let $n$ be a neutral and upper element of a nearlattice S.Define $\varphi: S \rightarrow(n] \times[n)$ by $\varphi(a)=(a \wedge n, a \vee n)$.

Then the following conditions are equivalent:

(i) $n$ is central;

(ii) $\varphi$ is an isomorphism.

Proof. $(i) \Rightarrow(i i) \quad \varphi(a)=(a \wedge n, a \vee n)$

$$
\begin{aligned}
\varphi(a \wedge b) & =((a \wedge b) \wedge n,(a \wedge b) \vee n) \\
& =((a \wedge n) \wedge(b \wedge n),(a \vee n) \wedge(b \vee n)) \\
& =(a \wedge n, a \vee n) \wedge(b \wedge n, b \vee n) \\
& =\varphi(a) \wedge \varphi(b)
\end{aligned}
$$

Similarly $\varphi(a \vee b)=\varphi(a) \vee \varphi(b)$

So $\varphi$ is homomorphism.

Now suppose $\varphi(a)=\varphi(b)$. This implies

$(a \wedge n, a \vee n)=(b \wedge n, b \vee n)$, and so, 
$a \wedge n=b \wedge n$ and $a \vee n=b \vee n$.

This implies $a=b$

Therefore $\varphi$ is one-one.

Let $t \in(n] \times[n)$.

Then $t=\left(t_{1}, t_{2}\right)$ such that $t_{1} \in(n], t_{2} \in[n)$.

Thus $t_{1} \leq n \leq t_{2}$.

Then there exists $r \in S$ such that $r \wedge n=t_{1}, r \vee n=t_{2}$.

This implies $t=(r \wedge n, r \vee n)=\varphi(r)$, and so $\varphi$ is onto.

Hence $S \cong(n] \times[n)$

(ii) $\Rightarrow($ i) . Let $a \leq n \leq b$

Since $\varphi$ is an isomorphism, so it is onto.

Then $(a, b) \in(n] \times[n)$.

Since $\varphi$ is onto, so there exists $r \in S$ such that $\varphi(r)=(a, b)$.

Thus $(r \wedge n, r \vee n)=(a, b)$, and so $r \wedge n=a, r \vee n=b$.

That is $r$ is the relative complement of $n$ in $[a, b]$.

Therefore $n$ is central $\square$

Thus we have the following results:

Theorem 1.10. Let $n$ be a central element of a nearlattice $S$. Then any $t \in S$ is central if and only if it is central in $S_{n}$.

Proof. By the above Lemma, $S \cong(n] \times[n)$

So $t \in S$ is central in $S$ if and only if it is also central in $(n] \times[n)$.

Then by Lemma 1.5. and Lemma 1.6, $t$ is central in $(n]^{d} \times[n)$.

As $t$ is central in $(n]^{d} \times[n)$ then by Lemma 1.5, it is central in $S_{n}$ as $(n]^{d} \times[n) \cong S_{n}$

Corollary 1.11. For a central element $n$ of a lattice $L, L$ is distributive and relatively complemented if and only if $L_{n}$ is so $\square$

Theorem 1.12. If $n$ is neutral in $L$, then the following conditions are equivalent:

(i) $n$ is central and $L$ is bounded;

(ii) There exists a unique $n^{\prime}$ such that for all $x \in L, x=m\left(n, x, n^{\prime}\right)$ 
(iii) $\left(L_{n} ; \cap, \cup\right)$ is a bounded lattice with $n^{\prime}$ as the largest element and for any $x, y \in L_{n}, x \cup y=m\left(x, n^{\prime}, y\right)$.

Proof. $($ i $) \Rightarrow$ (iii) follows from Theorem 1.3.

(iii) $\Rightarrow($ ii $)$. Since $x \cup y=m\left(x, n^{\prime}, y\right)$ for all $x, y \in L$ (ii) clearly follows by choosing $y=n$.

Moreover $n^{\prime}$ is unique as it is the largest element of $L_{n}$.

$$
\text { (ii) } \Rightarrow\left(\text { i). Clearly } n \wedge n^{\prime} \leq x \leq n \vee n^{\prime} \text { for all } x \in L\right. \text {. }
$$

Thus $L$ is bounded. Since $n$ is neutral, we already know by [2, Theorem 2.3.] that $L_{n}$ is a nearlattice with $n$ as the smallest element.

Now (ii) says that obviously $n^{\prime}$ is the largest element of $L_{n}$, and hence $L_{n}$ is a bounded lattice. Thus by Theorem 1.2, $n$ is central $\square$

Following result is due to Goetz [4].

Theorem 1.13. Let $\left(B ; \wedge, \vee,{ }^{\prime}, 0,1\right)$ be a Boolean algebra, $n \in B$. Then $B_{n}$ is also a Boolean algebra with $B_{n}=\left(B ; \cap, \cup,{ }^{\prime}, n, n^{\prime}\right)$.

Also the complement of any element is invariant under the formation of isotopes. Moreover $B \cong B_{n}$ 口

We conclude the paper with the following result which is an extension of the above result.

Theorem 1.14. Let $n$ be a central element of the distributive lattice $B=(B ; \wedge, \vee, 0,1)$. If the isotope $B_{n}=\left(B ; \cap, \cup,{ }^{\prime}, n, n^{\prime}\right)$ is the Boolean algebra, then $B$ is also Boolean algebra with the same complement.

Proof. By [1, Theorem 3.1.6], $B$ is distributive as $B_{n}$ is so.

Let $a^{\prime}$ be the complement of $a$ in $B_{n}$.

Then $a \cap a^{\prime}=n$ and $a \cup a^{\prime}=n^{\prime}$.

Then $\left(a \vee a^{\prime}\right) \wedge n=(a \wedge n) \vee\left(a^{\prime} \wedge n\right)$

$$
\begin{aligned}
& =\left(a \cap a^{\prime}\right) \wedge n \\
& =n \wedge n=n=1 \wedge n
\end{aligned}
$$

$n^{\prime}=a \cup a^{\prime}$

$=m\left(a, n^{\prime}, a^{\prime}\right)$

$=\left(a \vee n^{\prime}\right) \wedge\left(a \vee a^{\prime}\right) \wedge\left(a^{\prime} \wedge n^{\prime}\right)$

$1=n^{\prime} \vee n$ 


$$
\begin{aligned}
& =n \vee\left[\left(a \vee n^{\prime}\right) \wedge\left(a \vee a^{\prime}\right) \wedge\left(a^{\prime} \vee n^{\prime}\right)\right] \\
& =\left(a \vee n \vee n^{\prime}\right) \wedge\left(a \vee a^{\prime} \vee n\right) \wedge\left(a^{\prime} \vee n \vee n^{\prime}\right) \\
& =1 \wedge\left(a \vee a^{\prime} \vee n\right) \wedge 1 \\
& =a \vee a^{\prime} \vee n
\end{aligned}
$$

So, $a \vee a^{\prime} \vee n=1=1 \vee n$.

Thus $a \vee a^{\prime}=1$ (by the neutrality of $n$ ).

$$
\text { Again, } \begin{aligned}
0=\left(a \cap a^{\prime}\right) \wedge n^{\prime} & =\left[\left(a \wedge a^{\prime}\right) \vee(a \wedge n) \vee\left(a^{\prime} \wedge n\right)\right] \wedge n^{\prime} \\
& =\left(a \wedge a^{\prime} \wedge n^{\prime}\right) \vee\left(a \wedge n \wedge n^{\prime}\right) \vee\left(a^{\prime} \wedge n \wedge n^{\prime}\right) \\
& =\left(a \wedge a^{\prime} \wedge n^{\prime}\right) \vee o \vee o=a \wedge a^{\prime} \wedge n^{\prime}
\end{aligned}
$$

Thus $a \wedge a^{\prime} \wedge n^{\prime}=o \wedge n^{\prime}$

Again $a \cup a^{\prime}=n^{\prime}$

Thus $m\left(a, n^{\prime}, a^{\prime}\right)=n^{\prime}$ implies $\left(a \wedge a^{\prime}\right) \vee\left(a \wedge n^{\prime}\right) \vee\left(a^{\prime} \wedge n^{\prime}\right)=n^{\prime}$

This implies $\left(a \wedge a^{\prime}\right) \vee n^{\prime}=n^{\prime}=o \vee n^{\prime}$

Hence $a \wedge a^{\prime}=o$.

This implies that $a^{\prime}$ is also the complement of $a$ in $(B ; \wedge, \vee, o, 1)$.

Therefore $B$ is also Boolean $\square$

\section{REFERENCES}

1. Jahanara Begum, Study of n-ideals of a nearlattice by isotopes, Ph.D. Thesis, Jahangirnagar University, Savar, Dhaka, Bangladesh (2009).

2. Jahanara Begum and A.S.A. Noor, A ternary operation in a lattice, accepted in Ganit J. Bangladesh, Math. Soc.

3. W.H. Cornish and A.S.A.Noor, Around a neutral element of a nearlattice, comment. Math. Univ. Carolinae, 28(2) (1987).

4. A.Goetz, On Various Boolean Structures in a given Boolean algebra, Publ. Math. Debrecen 18(1971), 103-107.

5. M.G.Hossain and A.S.A.Noor, n-ideals of a nearlattice,J. Sc. The Rajshahi Univ. Studies, 28(2000), 105111.

6. Kolibiar, A ternary operation in lattices,Czechoslovak Math. J.6 (1956).

7. M.A. Latif and A.S.A.Noor, n-ideals of a lattice, The Rajshahi Univ.Studies (Part-B), 22(1994), 173-180.

8. A.S.A. Noor and M.A. Latif, Finitely generated n-ideals of a lattice, SEA Bull, Math. 22(1998), 73-79. 